\title{
Adjuvant Therapy for Pancreatic Cancer: A Logical Strategy in Search of Progress
}

\author{
Douglas B. Evans, $\mathrm{MD}^{1}$ and Robert A. Wolff, $\mathrm{MD}^{2}$ \\ ${ }^{1}$ Pancreatic Cancer Program, Medical College of Wisconsin, Milwaukee, WI; ${ }^{2}$ Department of Gastrointestinal Medical \\ Oncology, University of Texas M. D. Anderson Cancer Center, Houston, TX
}

In this issue of Annals of Surgical Oncology, Regine et al. ${ }^{1}$ report the long-term results of the U.S. Intergroup/ RTOG 9704 phase III trial of adjuvant therapy for surgically resected adenocarcinoma of the pancreas. In contrast to their initial analysis, they found no difference in survival between patients treated with either gemcitabine or 5-fluorouracil (5-FU) delivered before and after 5-FU-based chemoradiation., ${ }^{1,2}$ They offer the following possible explanations for the failure of the gemcitabine arm to be associated with a survival advantage: the frequent use of salvage gemcitabine therapy at the time of recurrence in those patients randomized to 5-FU; more advanced local disease (T3/T4) in the gemcitabine arm; the sequencing of chemoradiation, which interrupted potentially effective systemic therapy (rather than a chemotherapy followed by a chemoradiation sequencing strategy); and the delivery of only four cycles of gemcitabine rather than six, as in the CONKO-001 trial. ${ }^{3}$ Although these factors may have played a role, review of retrospective data and recently reported phase II and phase III trials (Tables 1 and 2) offers the following conclusions. ${ }^{3-5}$

Pancreaticoduodenectomy is a large operation and recovery is difficult. A surgery-first strategy with planned postoperative adjuvant therapy is by no means a guarantee that postoperative therapy will be delivered. Up to $50 \%$ of patients who undergo this operation will not receive intended therapy. ${ }^{6,7}$ This number may be higher if one mandates adjuvant therapy begin within eight weeks of surgery. In ESPAC-3 for example, enrollment occurred at an average rate of one patient per site per year, and even these highly selected patients often had difficulty tolerating

(C) Society of Surgical Oncology 2011

Published Online: 8 March 2011

D. B. Evans, MD

e-mail: devans@mcw.edu adjuvant therapy. ${ }^{4}$ By definition, all phase II and phase III adjuvant trials that enroll patients after recovery contain a large selection bias and involve only the most favorable patient subset. This reality of clinical trial design should be understood when comparing results from clinical trials that use alternative treatment schemas to include neoadjuvant therapy that places surgery after some form of induction therapy. In addition, such data represent a major drawback of conventional adjuvant treatment sequencing for pancreatic cancer because many patients do not receive multimodality therapy when surgery is performed first. ${ }^{8}$ As nonsurgical therapies become more effective, this disadvantage of a surgery-first treatment strategy will be become even more relevant.

In the absence of central review of preoperative imaging (to exclude those patients with obvious borderline resectable or locally advanced disease), there is currently no reliable and effective way to determine the completeness of surgical resection of the primary tumor. This presents a dilemma in solid tumor oncology that is unique to pancreas cancer; the AJCC Cancer Staging Manual outlines the importance of the SMA margin and its analysis, yet this recommendation is rarely followed. ${ }^{9}$ Surgeons do not consistently document the completeness of resection in operative dictations, and the pathologist, when faced with tumor at the inked margin (in those cases where the margin is correctly identified and inked), cannot tell the difference between an R1 and an R2 resection. ${ }^{10}$ Operative notes should not be signed off until the pathology report is available to allow for the $\mathrm{R}$ status of all cancer operations to be noted in the operative report.

Recently reported adjuvant trials likely contained a large number of patients with incompletely resected local disease, which contributed to the high rates of local recurrence in those trials that reported pattern-of-failure data. In addition, as a result of the modest rigor with which patients 


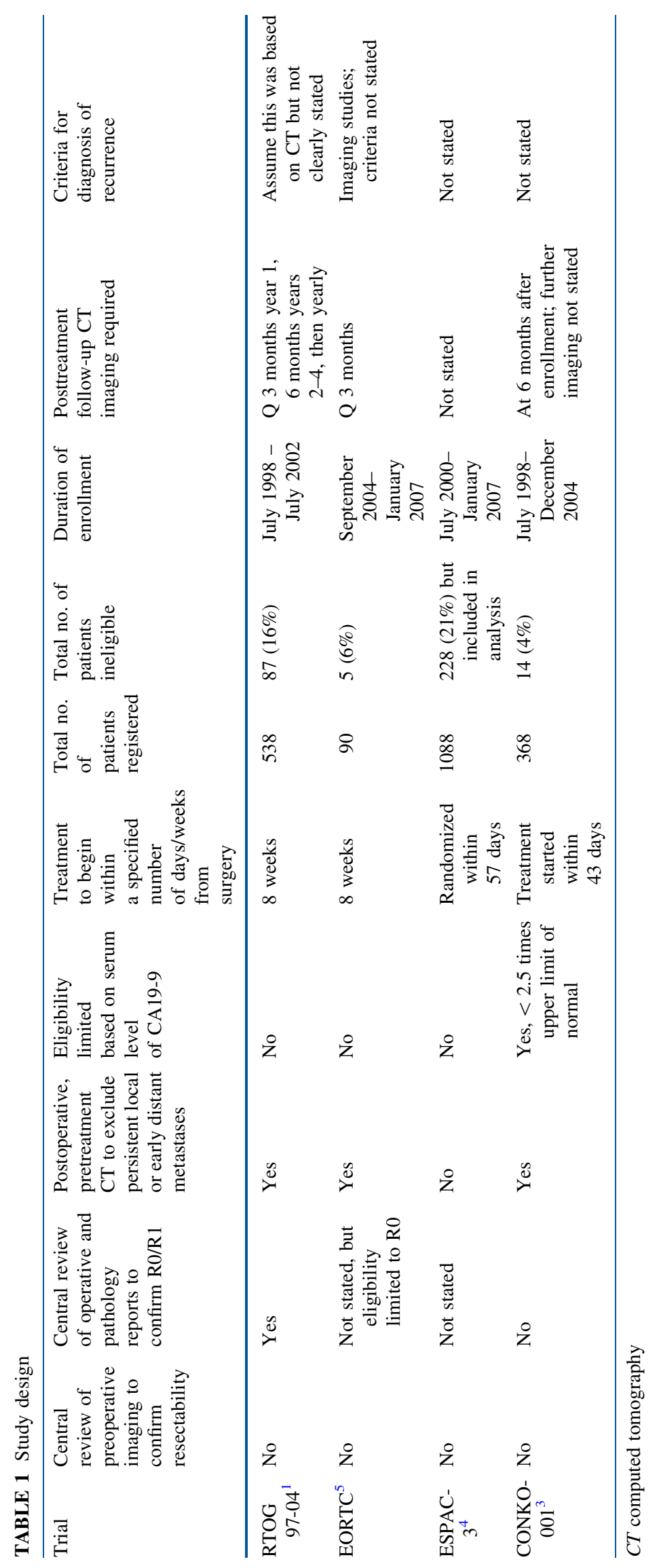




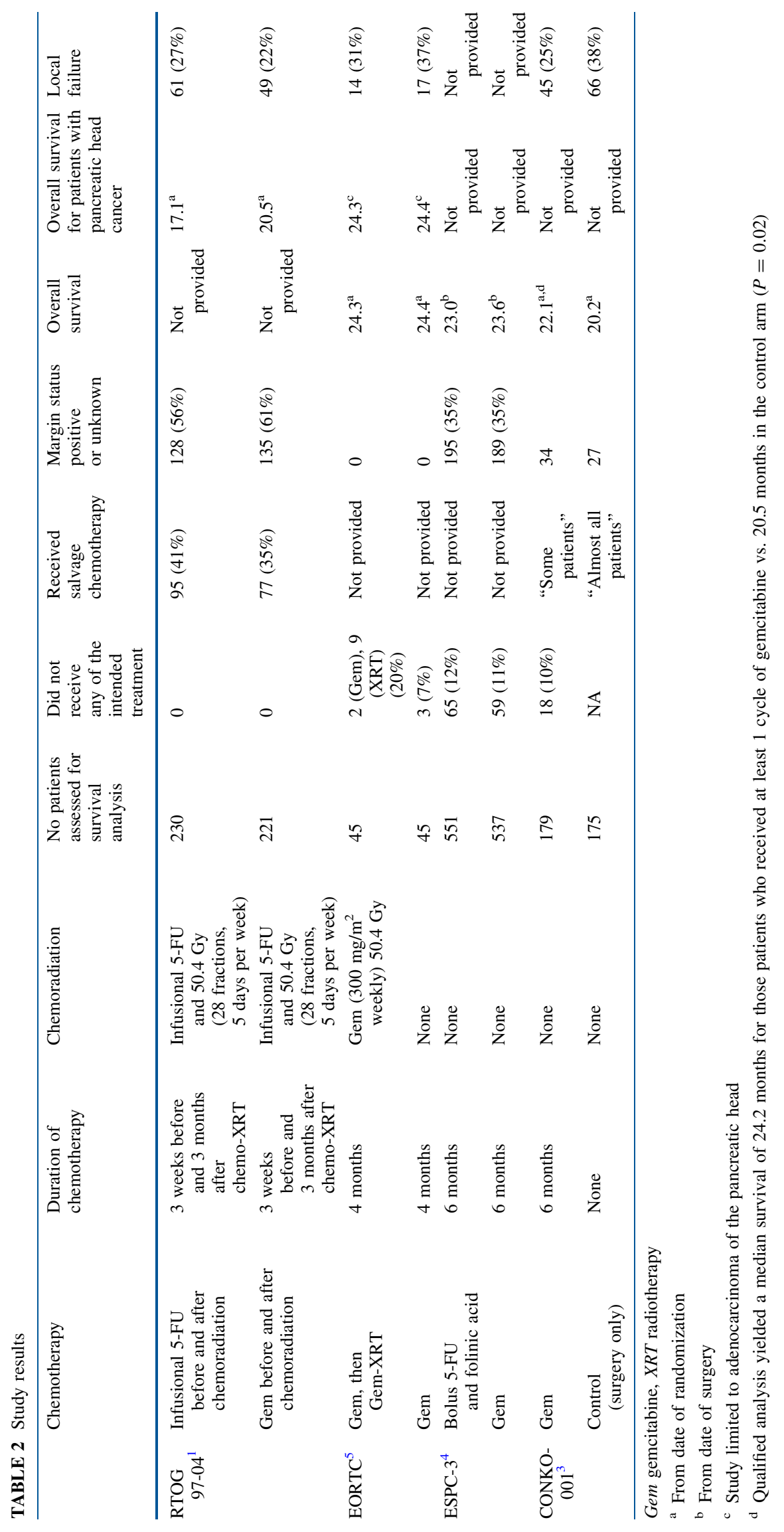


TABLE 3 Minimal criteria for the conduct of adjuvant therapy trials

- Central review of preoperative imaging to confirm that the primary tumor is resectable especially with reference to adjacent arteries

- Central review of operative and pathology reports to confirm a complete resection (R0/R1)

- Standardized system for the pathologic evaluation of the surgical specimen with specific reference to the SMA margin (or consensus for adhering to the system recommended in the AJCC Cancer Staging Manual)

- Central review of the histopathology

- Central review of postoperative/pretreatment CT to exclude persistent local or early distant metastases

- Standardized postoperative imaging for the detection of recurrent disease with CT-based definitions for local, regional, and distant recurrence

$C T$ computed tomography

were followed, the published rates of local recurrence should be viewed as minimums because they clearly underestimate the true frequency of recurrence. To what degree local disease affected quality and length of life is unknown. However, as systemic therapies improve, local recurrence may become more significant. Importantly, patients do die of "local-only" disease/recurrence; ignoring this important pattern of treatment failure is a mistake. $^{11}$

Given the differences in trial design and conduct outlined in Table 1, it is noteworthy that survival duration is quite similar between trials as shown in Table 2; any differences are likely of little clinical significance. Reasons for this may include the modest effectiveness of the systemic therapies applied; the relatively small number of patients destined to have local-only disease recurrence, which may have been favorably impacted by locoregional chemoradiation; and issues specific to treatment sequencing that uses a surgery-first strategy. Are the immunologic and systemic effects of such a large operation a negative influence (of high enough magnitude) on the ability to treat microscopic metastatic disease with currently available systemic agents? This is a difficult question to answer, but one that deserves more thoughtful attention, especially in light of recent reports of neoadjuvant treatment sequencing with much more favorable survival durations. ${ }^{12,13}$

For now, as we continue to struggle with the challenge of pancreatic cancer, it is imperative that the sophisticated audience of this journal take a leadership role in the standardization of the management of patients with localized pancreatic cancer. From imaging to local therapy, variability in treatment algorithms and quality of care (at specialty centers) should decrease. Table 3 outlines the minimum variables that should be controlled/mandated in current and future adjuvant therapy trials. At present, we need to transform from an attitude of giving an " $\mathrm{A}$ " for effort to those who complete a trial involving early-stage pancreatic cancer, to a position of demanding excellence in all components of the conduct of such trials to allow accurate interpretation of the results. Unfortunately, despite great effort to design a superior adjuvant trial, the current U.S. Intergroup trial-RTOG 0848 fails to incorporate all of the criteria in Table 3 into the surgery-first paradigm. ${ }^{14}$ For this disease, gaining experience with performing clinical trials may be as (or more) important than the question being asked. Current frustration with available therapies should be replaced with an energy for innovative trial design and standardized trial methodology.

\section{REFERENCES}

1. Regine WF, Winter KA, Abrams R, et al. Fluorouracil-based chemoradiation with either gemcitabine or fluorouracil chemotherapy after resection of pancreatic adenocarcinoma: 5-year analysis of the US Intergroup/RTOG 9704 phase III Trial. Ann Surg Oncol. doi:10.1245/s10434-011-1630-6.

2. Regine W, Winter K, Abrams R, et al. Fluorouracil vs gemcitabine chemotherapy before and after fluorouracil-based chemoradiation following resection of pancreatic adenocarcinoma. J Am Med Assoc. 2008; 299:1019-26.

3. Oettle H, Post S, Neuhaus P, et al. Adjuvant chemotherapy with gemcitabine vs observation in patients undergoing curative-intent resection of pancreatic cancer. JAMA. 2007;297:267-77.

4. Neoptolemos J, Stocken D, Bassi C, et al. Adjuvant chemotherapy with fluorouracil plus folinic acid vs gemcitabine following pancreatic cancer resection. JAMA. 2010;304:1073-81.

5. Van Laethem J, Hammel P, Mornex F, et al. Adjuvant gemcitabine alone versus gemcitabine based chemoradiotherapy after curative resection for pancreatic cancer: A randomized EORTC40013-22012/FFCD-9203/GERCOR phase II study. J Clin Oncol. 2010;28:4450-6.

6. Herman J, Swartz, Hsu C, et al. Analysis of fluorouracil-based adjuvant chemotherapy and radiation after pancreaticoduodenectomy for ductal adenocarcinoma of the pancreas: Results of a large, prospectively collected database and the Johns Hopkins Hospital. J Clin Oncol. 2008;26:3503-10.

7. Simons J, Chau Ng S, McDade T, et al. (2010) Progress for resectable cancer? A population-based assessment of US practices. Cancer 116: 2503.

8. Wolff R, Varadhachary G, Evans D. Adjuvant therapy for adenocarcinoma of the pancreas: Analysis of reported trials and recommendations for future progress. Ann Surg Oncol. 2008;15: 2773-86.

9. Edge S, Byrd D, Compton C, Fritz A, Greene F, Trotti A III, editors. American Joint Committee on Cancer. AJCC cancer staging manual. 7th ed. New York: Springer-Verlag; 2010; 24:241-9.

10. Katz MH, Merchant N, Brower S, et al. Standardization of surgical and pathologic variables is needed in multicenter trials of adjuvant therapy for pancreatic cancer results from the ACOSOG Z5031 trial. Ann Surg Oncol. 2010;18:337-44. 
11. Iacobuzio-Donahue C, Fu B, Yachida S, et al. DPC4 gene status of the primary carcinoma correlates with patterns of failure in patients with pancreatic cancer. J Clin Oncol. 2009;27:1806-13.

12. Evans D, Varadhachary G, Crane C, et al. Preoperative gemcitabine-based chemoradiation for patients with resectable adenocarcinoma of the pancreatic head. J Clin Oncol. 2008;26: 3496-502.

13. Varadhachary G, Wolff R, Evans D, et al. Preoperative gemcitabine and cisplatin followed by gemcitabine-based chemoradiation for resectable adenocarcinoma of the pancreatic head. J Clin Oncol. 2008;26:3487-95.

14. US National Institutes of Health. Gemcitabine hydrochloride with or without erlotinib hydrochloride followed by the same chemotherapy regimen with or without radiation therapy and capecitabine or fluorouracil in treating patients with pancreatic cancer that has been removed by surgery. February 15, 2011. http://www.clinicaltrials.gov/ct2/show/NCT01013649?term=Pan creatic + Cancer + and + RTOG\&rank $=6$. 